\title{
Spin correlations in a quasi-one-dimensional electron gas
}

\author{
B. Tanatar \\ Department of Physics, Bilkent University, Bilkent, 06533 Ankara, Turkey \\ Received 2 April 1996
}

\begin{abstract}
We study the spin correlations in a quasi-one-dimensional electron gas within the self-consistent-field approximation (SCFA). Electrons confined in a parabolic potential restricted to have free motion in one space dimension, and interact via a Coulomb-type potential are assumed to model quantum wires as realized in semiconductor structures. Density and spin-density response of the interacting electron system is investigated where correlation effects beyond the randomphase approximation (RPA) are embodied in the local-field factors. We calculate the spin-dependent pair-correlation functions and effective potentials, and determine the paramagnon dispersion. We find that electron correlation effects signal a paramagnetic instability for $r_{\mathrm{s}}>1.5$.
\end{abstract}

Keywords: Spin correlations; Q1DEG

\section{Introduction}

Quasi-one-dimensional (Q1D) electron systems as they occur in semiconducting structures, in which the electrons are confined to move freely only in one space dimension, is a subject of continuing interest. Advances in growth techniques and nanometerscale semiconductor device fabrication, have made it possible to manufacture Q1D systems with many interesting experimental results. The main motivation for studying these low-dimensional systems comes from their technological potential such as high-speed electronic devices and quantum-wire lasers. Other than the practical implications, electrons in Q1D structures offer an interesting manybody system for condensed-matter theories.

In this paper we study the response of a Q1D electron system to a weak external magnetic field, namely the wave vector- and frequency-dependent paramagnetic susceptibility. We assume that the
Q1D electrons are embedded in a uniform positive background to maintain charge neutrality. We treat the electron system as a Fermi liquid, i.e., with a well-defined Fermi surface at zero temperature and interaction via Coulomb potential, which seems to be supported by the experimental observations $[1,2]$ of collective excitations in GaAs quantum wires. It is believed that even though the Q1D electrons are not strictly Fermi liquids, the finite temperature and disorder effects restore such a picture [3]. There are well-established theoretical results [4] which predict non-Fermi liquid type (i.e., Tomonaga-Luttinger liquids) properties for an interacting one-dimensional (1D) electron system. In view of the fact that most Tomonaga-Luttinger liquid calculations use a contact interaction between the electrons, we feel that a realistic (longrange) Coulomb interaction model should be relevant to the understanding of semiconductor based quantum wire structures [5]. 
The enhancement of the paramagnetic susceptibility of an interacting electron system over its Pauli value is due to short-range Coulomb and exchange effects. We investigate the correlation effects within the self-consistent field approximation (SCFA) of Singwi et al. [6]. The density and spin-density responses are combined to determine the spin-dependent pair-correlation functions and effective potentials in the system. The dispersion relation for collective excitations of spin fluctuations (paramagnons) is obtained. We find that the Q1D electron system shows a paramagnetic instability at low densities, as in the $2 \mathrm{D}$ and $3 \mathrm{D}$ electron gas cases [7-9].

Although a fair amount of literature [10-14] exists on the electronic correlations in quantum wire structures, studies on the spin correlations are largely neglected. Spin correlations in a 3D electron system within the SCFA was first studied by Lobo et al. [15]. Diagrammatic approaches employing ladder sums were utilized to treat accurately the short-range part of the Coulomb interaction $[16,17]$. The correlation effects in a Q1D electron system within the STLS scheme were studied using different wire models $[10,11]$.

The rest of this paper is organized as follows. In Section 2 we outline the method of SCFA for density and spin-density responses. We introduce a quantum wire model. Our results for static and magnetic structure factors, spin-dependent paircorrelation functions and effective potentials are presented in Section 3. We discuss the paramagnon dispersion predicted by our calculations, and the onset of paramagnetic instability at low density. We conclude with a brief summary.

\section{Theory}

In the mean-field approximation $[6,15]$, the wave vector- and frequency-dependent density and spindensity response functions are expressed in the form

$\chi^{\mathrm{d}}(q, \omega)=\frac{\chi_{0}(q, \omega)}{1-\psi^{\mathrm{s}}(q) \chi_{0}(q, \omega)}$

and

$\chi^{\mathrm{s}}(q, \omega)=-g^{2} \mu_{\mathrm{B}}^{2} \frac{\chi_{0}(q, \omega)}{1-\psi^{\mathrm{a}}(q) \chi_{0}(q, \omega)}$, where $\chi_{0}(q, \omega)$ is the $1 \mathrm{D}$ free-electron polarizability $[12] . \psi^{s}(q)$ and $\psi^{\mathrm{a}}(q)$ are, respectively, the spin-symmetric and spin-antisymmetric potentials, $g$ is the Lande factor, and $\mu_{\mathbf{B}}$ is the Bohr magneton. The fluctuation-dissipation theorem enables us to write the static structure factor $S(q)$ and the magnetic structure factor $\tilde{S}(q)$ in terms of the response functions, viz.,

$S(q)=-\frac{1}{n \pi} \int_{0}^{\infty} \mathrm{d} \omega \chi^{\mathrm{d}}(q, \mathrm{i} \omega)$

$\tilde{S}(q)=\frac{1}{n \pi g^{2} \mu_{\mathrm{B}}^{2}} \int_{0}^{x} \mathrm{~d} \omega \chi^{\mathrm{s}}(q, i \omega)$,

where the frequency integrations are to be performed along the imaginary axis to better capture the collective mode contributions. The effective potentials, in the mean-field approximation, are given by $\psi^{\mathrm{s}}(q)=V(q)[1-G(q)]$, and $\psi^{\mathrm{a}}(q)=V(q) I(q)$, where $V(q)$ is the Q1D Coulomb potential. $G(q)$ and $I(q)$ are the static local-field factors arising from the short-range Coulomb correlations and the exchange-correlation effects for the density and spindensity responses, respectively. In the approximation scheme of Singwi et al. (STLS), they are given, respectively, as $[6,15]$

$$
\begin{aligned}
& G(q)=-\frac{1}{n} \int_{-\infty}^{\infty} \frac{\mathrm{d} k}{2 \pi} \frac{k V(k)}{q V(q)}[S(q-k)-1], \\
& I(q)=\frac{1}{n} \int_{-\infty}^{\infty} \frac{\mathrm{d} k}{2 \pi} \frac{k V(k)}{q V(q)}[\tilde{S}(q-k)-1],
\end{aligned}
$$

where $n$ is the linear electron density. In terms of the Fermi wave vector we have $n=2 k_{\mathrm{F}} / \pi$. The electron gas parameter is defined as $r_{\mathrm{s}}=\pi /\left(4 k_{\mathrm{F}} a_{\mathrm{B}}\right)$, in which $a_{\mathrm{B}}=\varepsilon_{0} /\left(e^{2} m^{*}\right)$ is the effective Bohr radius in the semiconducting wire with background dielectric constant $\varepsilon_{0}$ and electron effective mass $m^{*}$.

The model we use in our calculation for the Q1D electron system is developed by $\mathrm{Hu}$ and O'Connell [13], and is applicable to the experimental realizations of semiconducting systems. The change carriers are assumed to be in a zero thickness $x y$-plane with a harmonic confinement potential in the $y$-direction so that the subband energies are $\varepsilon_{n}=\Omega\left(n+\frac{1}{2}\right)$ where $\Omega$ describes the strength of the confining potential. The Coulomb interaction between particles in the lowest subband within this 
model is given by $V(q)=\left(2 e^{2} / \varepsilon_{0}\right) F(q)$, where $F(q)=\frac{1}{2} \exp \left(b^{2} q^{2} / 4\right) K_{0}\left(b^{2} q^{2} / 4\right)$ in which $K_{0}(x)$ is the modified Bessel function. The characteristic length $b=1 /(m \Omega)^{1 / 2}$ is related to the confining potential and gives a measure of the lateral width of the quantum wire. The large $q$ limit of the parabolic confinement model gives $F(q) \rightarrow(\pi / 2)^{1 / 2} /(b q)$. For long wavelengths, viz., $q \rightarrow 0$, all models behave as $\sim \ln (x q)$, where $x$ is some length parameter, depending on the Q1D model used. The weak logarithmic $q$ dependence is a result of the effective Coulomb interaction between the charge carriers in a Q1D structure, and is essentially independent of the model describing them $[14,18]$.

Once the self-consistency for the static structure factors are reached, we can evaluate the spin symmetric and spin-antisymmetric pair-correlation functions by the Fourier transforms

$g(r)=1+\frac{1}{2} \int_{0}^{\infty} \mathrm{d} q \cos (q r)[S(q)-1]$,

$\tilde{g}(r)=\frac{1}{2} \int_{0}^{\infty} \mathrm{d} q \cos (q r)[\tilde{S}(q)-1]$.

These expressions yield the analytical results in the Hartree-Fock approximation

$$
\begin{aligned}
& g_{\mathrm{HF}}(r)=1-\frac{(1-\cos (2 r))}{4 r^{2}} \text { and } \\
& \tilde{g}_{\mathrm{HF}}(r)=-\frac{(1-\cos (2 r))}{4 r^{2}} .
\end{aligned}
$$

\section{Results and discussion}

We solve the above set of equations [Eqs. (1)-(6)] that describe the structure factors and local-field corrections for density and spin-density response self-consistently. The numerical accuracy attained is typically $0.01 \%$. In Fig. 1 we show the magnetic structure factor $\tilde{S}(q)$ for different values of $r_{\mathrm{s}}$, in a quantum wire with width parameter $b=2 a_{\mathrm{B}}$. The dotted, dashed, and solid lines are for $r_{\mathrm{s}}=0.5,1$, and 1.5 , respectively. We point out that for small values of $r_{\mathrm{s}}$, the magnetic structure factor is very similar to the Hartree-Fock result where the electron gas is treated as a free-electron system. As the

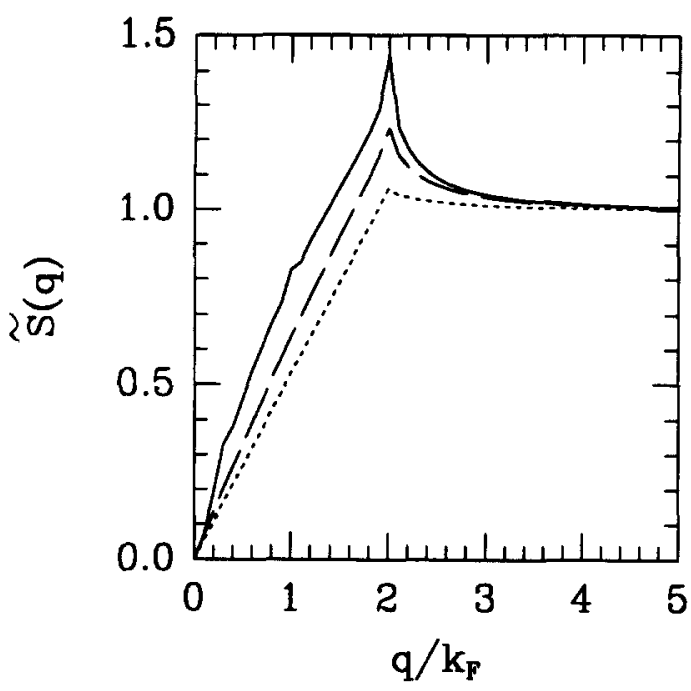

Fig. 1. The magnetic structure factor $\tilde{S}(q)$ in the SCFA for $r_{\mathrm{s}}=0.5$ (dotted line), $r_{\mathrm{s}}=1$ (dashed line), and $r_{\mathrm{s}}=1.5$ (solid line). We take the quantum wire width parameter $b=2 a_{\mathrm{B}}$.

density is decreased (increasing $r_{\mathrm{s}}$ ), a sharp peak in $\tilde{S}(q)$ develops at $q=2 k_{\mathrm{F}}$. It is interesting to note that such a singular behavior is also present in Hubbard model calculations [19]. It becomes exceedingly difficult to obtain self-consistent solutions for $r_{\mathrm{s}}>1.5$. Similar behavior of the self-consistent equations in a $2 \mathrm{D}$ electron gas was argued to imply an instability developing in the system [7]. Divergence in the spin-response of a $2 \mathrm{D}$ electron gas was also found by Yarlagadda and Giuliani [8] in various approximations. More elaborate theories of spin correlations in 3D seem to indicate the existence of instability at a much lower density (i.e., high $r_{\mathrm{s}}$ ) $[20,21]$. We believe that the instability predicted by the SCFA needs to be explored by theories that go beyond the present mean-field approach. We also note that if material parameters for GaAs are used, the critical density around which the instability occurs is obtained to be $n \sim 10^{4} \mathrm{~cm}^{-1}$. This value of density is within the range of present technology of semiconducting quantum wires. Experimental investigations in this direction should be interesting.

The $\tilde{S}(q)$ in different approximations is shown in Fig. 2. In the random-phase approximation (RPA) there is no local-field correction, i.e., $G(q)=0$. A simplified attempt to go beyond the RPA is 


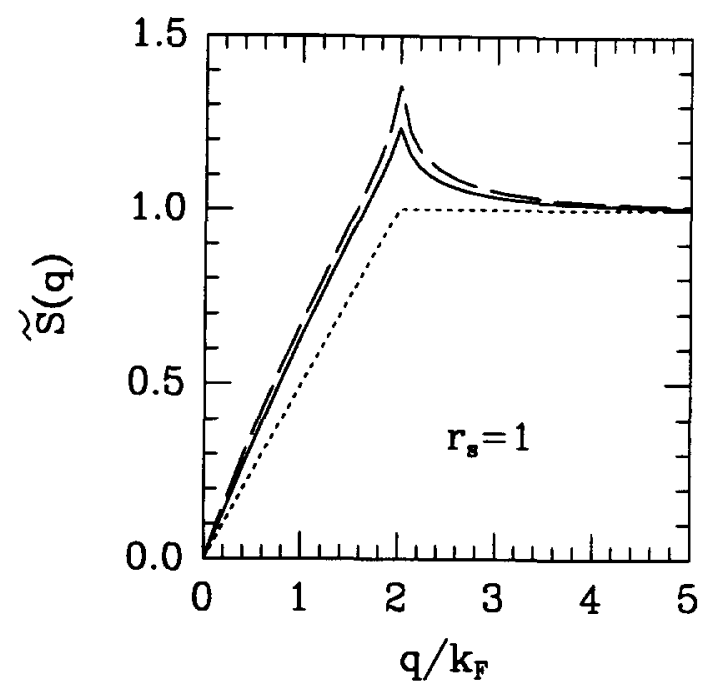

Fig. 2. The magnetic structure factor $\tilde{S}(q)$ at $r_{\mathrm{s}}=1$ in different approximations. Solid, dashed, and dotted lines indicate the SCFA, Hubbard approximation, and HFA, respectively.

provided by the Hubbard approximation (HA) in which the Pauli hole around electrons is taken into account. The one-dimensional Hubbard approximation using our parabolic confinement model is expressed as

$I_{\mathrm{H}}(q)=-\frac{1}{2} \frac{V\left(\sqrt{q^{2}+k_{\mathrm{F}}^{2}}\right)}{V(q)}$.

As in the $2 \mathrm{D}$ case [7], $\tilde{S}_{\mathbf{H}}(q)$ (dashed line in Fig. 2 ) exhibits a sharper peak at $q=2 k_{\mathrm{F}}$ than the selfconsistent result $\tilde{S}(q)$.

Collective excitations in an electron gas, may be studied as complex poles of the density and spindensity response functions $\chi^{\mathrm{d} . \mathrm{s}}(q, \omega)$, or as peaks of the dynamic structure factor $S(q, \omega)$. The calculation of the collective spin modes is similar to that of density excitations [14], and we find

$\omega_{q}^{2}=\frac{\omega_{+}^{2}-\omega_{-}^{2} \mathrm{e}^{-A(q)}}{1-\mathrm{e}^{-A(q)}}$,

where $\omega_{ \pm}=\left|q^{2} / 2 m \pm q k_{\mathrm{F}} / m\right|, \quad$ and $\quad A(q)=$ $\pi^{2} q /\left[8 r_{\mathrm{s}} F(q) I(q)\right]$. We show the dispersion $\omega_{q}$, of the paramagnon peak in Fig. 3 as a function of $q$, at $r_{\mathrm{s}}=1$. Actually, as $q \rightarrow 0, \omega_{q}$ is independent of $r_{\mathrm{s}}$ because of $I(q)$ which we discuss later. For small $q, \omega_{q}$ shows a linear behavior, reminiscent of phe-

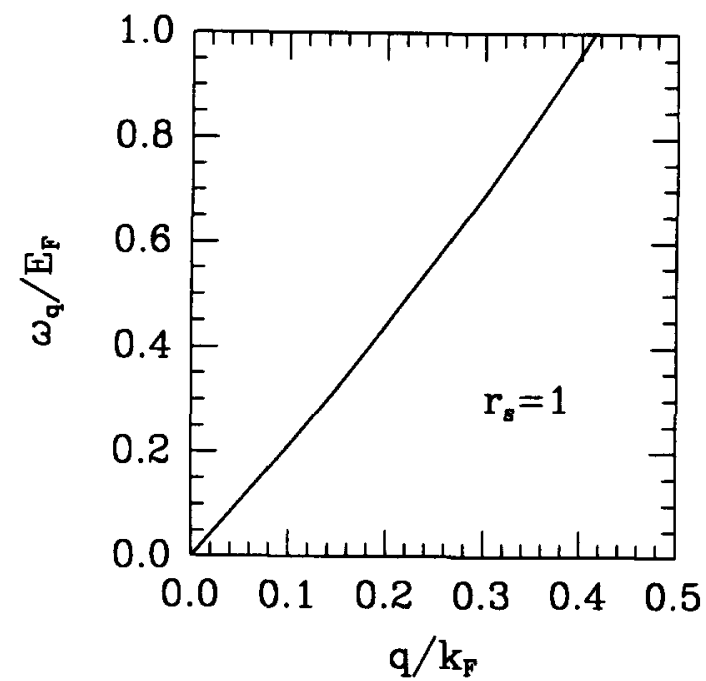

Fig. 3. The dispersion of the paramagnon peak as a function of $q$ at $r_{\mathrm{s}}=1.5$.

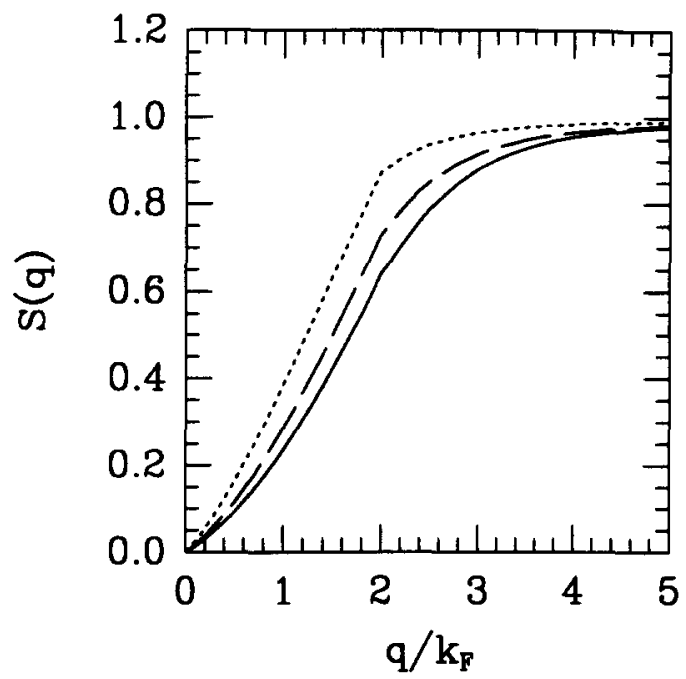

Fig. 4. The static structure factor $S(q)$ in the SCFA for $r_{s}=1$ (dotted line), $r_{\mathrm{s}}=2$ (dashed line), and $r_{\mathrm{s}}=3$ (solid line). We take the quantum wire width parameter $b=2 a_{\mathrm{B}}$.

nomenological models, (i.e., Stoner model) in which $I(q)$ is constant.

The self-consistent solutions are easy to obtain for the static structure factor $S(q)$ even for larger values of $r_{\mathrm{s}}$. We plot $S(q)$ for various values of $r_{\mathrm{s}}$ in Fig. 4. Density correlations in Q1D electron 


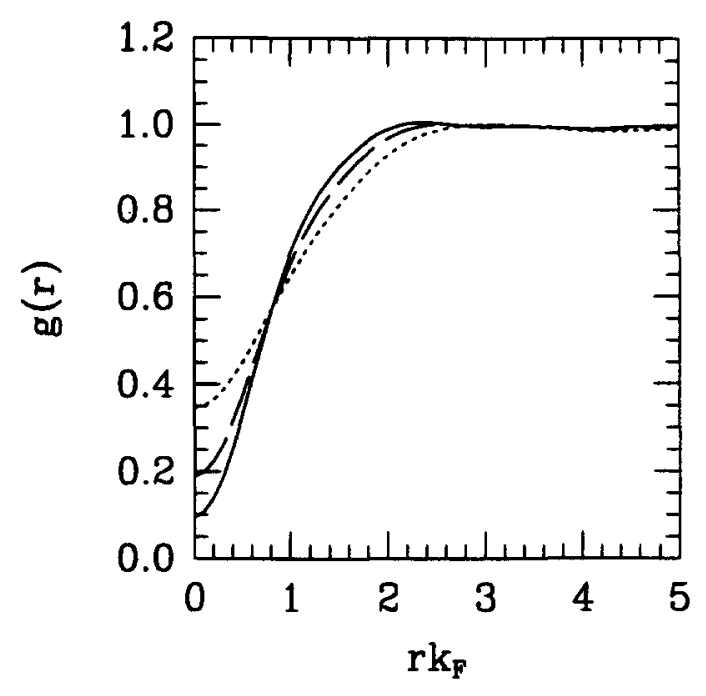

Fig. 5. The pair-correlation function $g(r)$ obtained by Fourier transform of $S(q)$, for $r_{\mathrm{s}}=1$ (dotted line), $r_{\mathrm{s}}=2$ (dashed line), and $r_{s}=3$ (solid line).

systems with different wire models were studied earlier $[10,11]$. Our results are in qualitative agreement with such calculations. The structure factors $S(q)$ and $\tilde{S}(q)$ thus calculated are used to obtain the spin-symmetric and spin-antisymmetric pair-cor- relation functions. We show the probability of finding an electron at $r$ if another electron is located at the origin (irrespective of their spins) in Fig. 5. The pair-correlation function $g(r)$ is the Fourier transform of $S(q)$ shown in Fig. 4. We notice that for small $r, g(r)$ gets smaller, as $r_{\mathrm{s}}$ increases. We find that for $r_{\mathrm{s}}>3, g(0)$ becomes negative, a drawback of the STLS scheme, known to exist also in 2D and $3 \mathrm{D}$.

The following combinations of the pair-correlation functions

$g_{\Uparrow}(r)=g(r)+\tilde{g}(q)$ and $g_{\downarrow}(r)=g(r)+\tilde{g}(q)$,

determine the spin-dependent pair correlations. We display the resulting $g_{\Uparrow}(r)$ and $g_{\downarrow}(r)$ in Figs. 6(a) and (6b), respectively. It is evident from Fig. 6(a) that $g_{\uparrow}(r)$ is largely independent of $r_{\mathrm{s}}$, and its value at zero separation increases with increasing $r_{s}$.

The spin-symmetric and anti-symmetric effective potentials (in units of $V(q)$ ) are displayed in Figs. 7(a) and (7b) for several densities. We observe that correlation effects increase with increasing $r_{\mathrm{s}}$. The limiting behaviours of $\psi^{\mathrm{s}}(q)$ and $\psi^{\mathrm{a}}(q)$ are largely independent of $r_{\mathrm{s}}$ as $q \rightarrow 0$, since correlation effects become negligible at large distances. Using the expressions for the density and spin-density
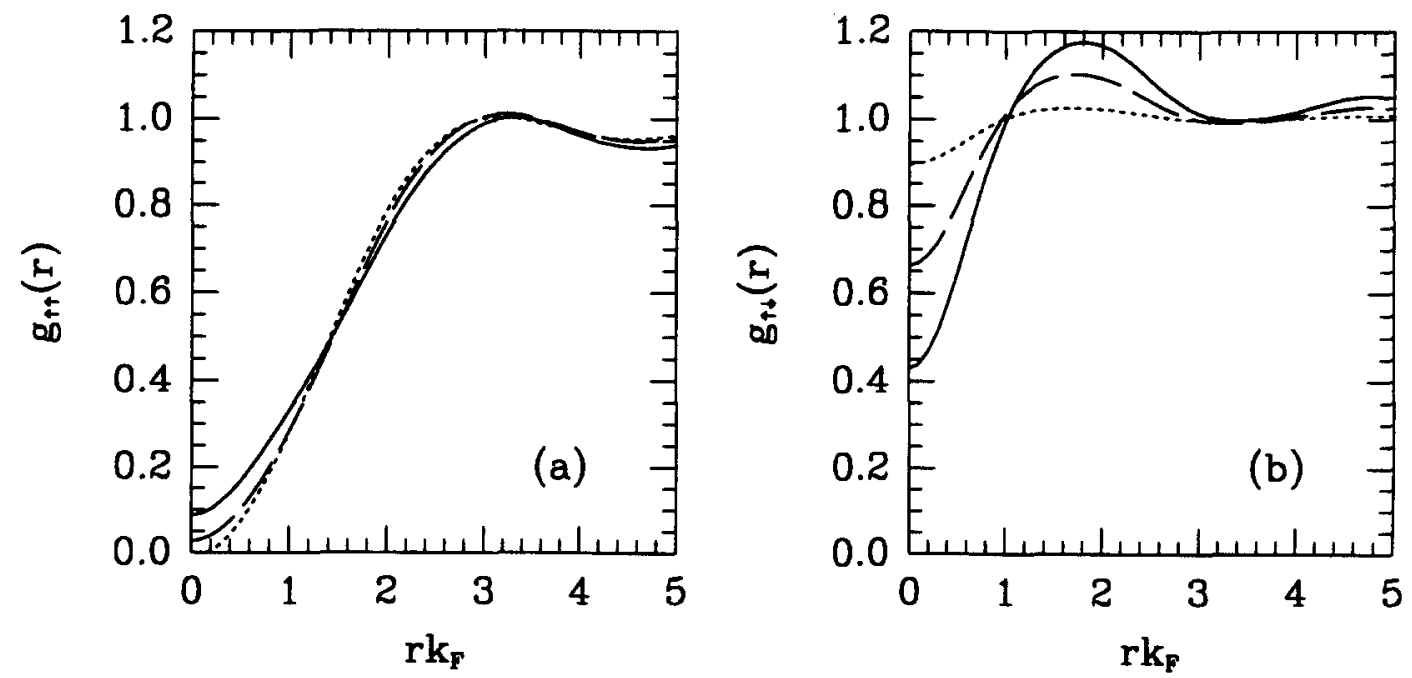

Fig. 6. The spin-dependent pair-correlation functions (a) $g_{\pi}(r)$, and (b) $g_{\uparrow l}(r)$ obtained from $g(r)$ and $\tilde{g}(r)$, for $r_{\mathrm{s}}=0.5$ (dotted line), $r_{\mathrm{s}}=1$ (dashed line), and $r_{\mathrm{s}}=1.5$ (solid line). 

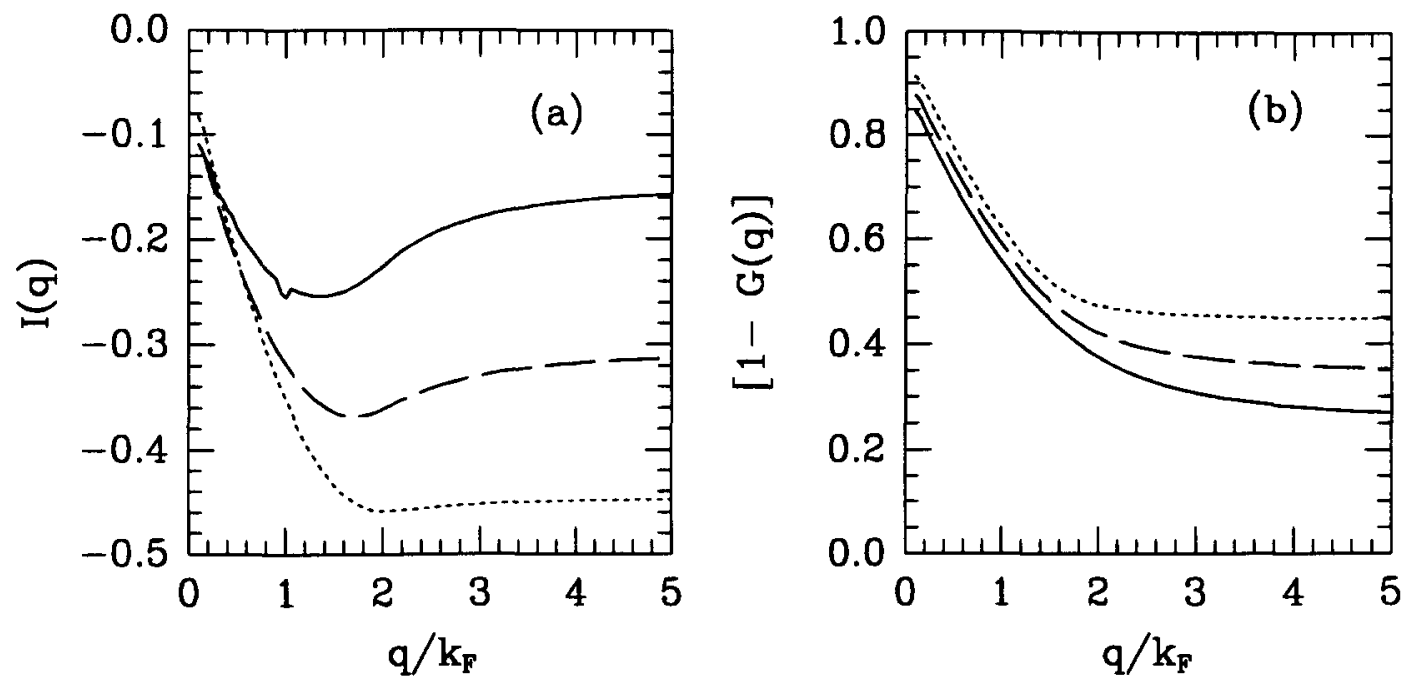

Fig. 7. (a) The spin-symmetric and (b) spin-antisymmetric effective potentials as a function of $q$, for $r_{\mathrm{s}}=0.5$ (dotted line), $r_{\mathrm{s}}=1$ (dashed line), and $r_{\mathrm{s}}=1.5$ (solid line).

local-field corrections and the asymptotic form for the Coulomb potential, we obtain

$\lim _{q \rightarrow 0} \psi^{\mathrm{s}}(q) \rightarrow \frac{2 e^{2}}{\varepsilon_{0}}[\ln (q b / 2)+\gamma]$

$\lim _{q \rightarrow 0} \psi^{\mathrm{a}}(q) \rightarrow-\frac{2 e^{2}}{\varepsilon_{0}} \gamma$

where

$\gamma=-\frac{1}{2} \int_{0}^{\infty} \mathrm{d} k F(k)[S(k)-1]$.

In the opposite limit, viz., $q \rightarrow \infty$, we find

$\lim _{q \rightarrow 0} \psi^{s}(q) \rightarrow \frac{2 e^{2}}{\varepsilon_{0}}(\pi / 2)^{1 / 2} g(0) / q b$,

$\lim _{q \rightarrow 0} \psi^{\mathrm{a}}(q) \rightarrow \frac{2 e^{2}}{\varepsilon_{0}}(\pi / 2)^{1 / 2} \tilde{g}(0) / q b$,

where $g(0)$ and $\tilde{g}(0)$ are, respectively, the spin-symmetric and spin-antisymmetric pair correlation functions at $r=0$. Some exact limiting expressions for the local-field factors in Q1D electron systems have recently been discussed by Fantoni and Tosi [22]. The spin-dependent potentials $\psi_{\uparrow}$ and $\psi_{\uparrow \downarrow}$ calculated by

$\psi_{\Uparrow}(q)=\psi^{\mathrm{s}}(q)+\psi^{\mathrm{a}}(q)$ and

$\psi_{\uparrow \downarrow}(q)=\psi^{\mathrm{s}}(q)-\psi^{\mathrm{a}}(q)$,

are shown in Figs. 8(a) and 8(b), respectively. We find that the magnitude of $\psi_{\uparrow_{t}}(q)$ is always greater than that of $\psi_{\uparrow}(q)$ for a given $r_{\mathrm{s}}$, since the charge depletion for a pair of electrons with parallel spins is more than that for a pair with antiparallel spins. The fact that $g_{\uparrow}(r)$ is mostly independent of $r_{\mathrm{s}}$ is also reflected in the behavior of $\psi_{\uparrow}(q)$.

The wave-vector-dependent spin susceptibility within the SCFA is readily obtained from Eq. (2) after setting $\omega=0$,

$\chi^{\mathrm{S}}(q)=g^{2} \mu_{\mathrm{B}}^{2} \frac{\rho\left(\varepsilon_{\mathrm{F}}\right) k_{\mathrm{F}}}{q} \frac{\ln \left|\frac{q+2 k_{\mathrm{F}}}{q-2 k_{\mathrm{F}}}\right|}{1+\frac{16 r_{k}, k_{\mathrm{F}}}{\pi^{2} q} F(q) I(q) \ln \left|\frac{q+2 k_{\mathrm{F}}}{q-2 k_{\mathrm{F}}}\right|}$,

where $\rho\left(\varepsilon_{\mathrm{F}}\right)=2 m /\left(\pi k_{\mathrm{F}}\right)$ is the density of states of $1 \mathrm{D}$ electrons at the Fermi surface. In the above, we have also made use of the explicit form of $\chi_{0}(q)$, the static susceptibility of the free electron gas. $\chi_{0}(q)$ has a singularity at $q=2 k_{\mathrm{F}}$, leading to the well-known Peierls instability. The SCFA removes this singular behavior and $\chi^{\mathrm{s}}(q)$ remains sharp but finite at $q=2 k_{\mathrm{F}}$ with the numerical value $\chi^{\mathrm{s}}\left(2 k_{\mathrm{F}}\right)=$ 

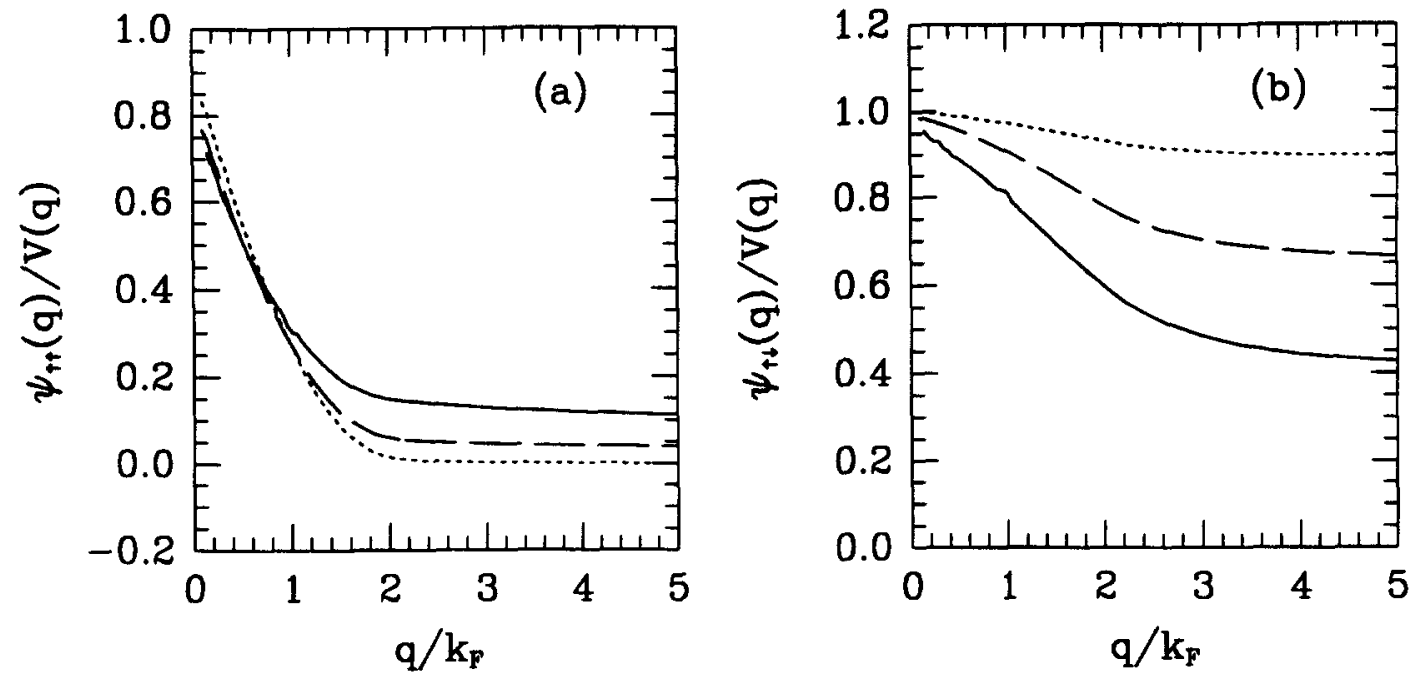

Fig. 8. The spin-dependent effective potentials (a) $\psi_{\pi}(q)$, and (b) $\psi_{\dagger \downarrow}(q)$ for $r_{s}=0.5$ (dotted line), $r_{s}=1$ (dashed line), and $r_{\mathrm{s}}=1.5$ (solid line).

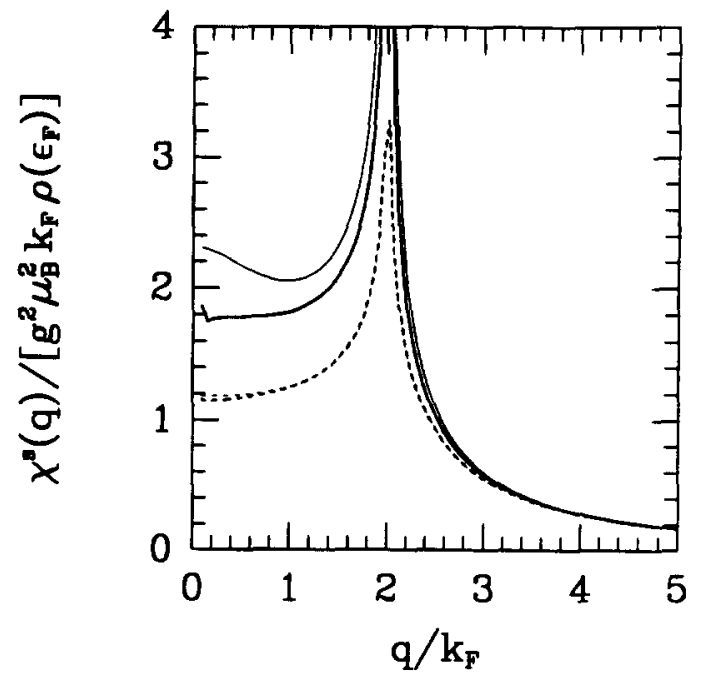

Fig. 9. The static spin response $\chi^{*}(q)$ as a function of $q$, for $r_{\mathrm{s}}=0.5$ (dashed lines) and $r_{\mathrm{s}}=1$ (solid lines). Thick and thin lines indicate the SCFA and Hubbard approximation results, respectively.

$g^{2} \mu_{\mathrm{B}}^{2} \pi^{2} k_{\mathrm{F}} \rho\left(\varepsilon_{\mathrm{F}}\right) /\left[16 r_{\mathrm{s}} F\left(2 k_{\mathrm{F}}\right) I\left(2 k_{\mathrm{F}}\right)\right]$. In Fig. 9 we show $\chi^{\mathrm{s}}(q)$ for $r_{\mathrm{s}}=0.5$ (dashed), and 1 (solid), in the SCFA (thick lines) and the Hubbard approximation (thin lines). We observe that for $r_{\mathrm{s}}=0.5$, the difference between SCFA and HA is very small, and as $r_{\mathrm{s}}$ increases, the peak at $q=2 k_{\mathrm{F}}$ becomes sharper.

\section{Summary}

In summary, we have studied the spin-correlations in a Q1D electron system interacting via Coulomb potential in the self-consistent scheme of Singwi et al. [6, 15]. Our SCFA calculation predicts a paramagnetic instability for lower densities such that $r_{\mathrm{s}}>1.5$. Treated as a Fermi system the static properties of the Q1D electron gas as occurring in semiconducting quantum wires shows qualitatively similar behavior found in 2D and 3D cases. Our results should be qualitatively the same for different models of quantum wire structures.

\section{Acknowledgements}

This work is partially supported by the Scientific and Technical Research Council of Turkey (TUBITAK) under Grant No. TBAG-AY/77. We thank Prof. G. Senatore for useful discussions. 


\section{References}

[1] A.R. Goñi, A. Pinczuk, J.S. Weiner, J.M. Calleja, B.S. Dennis, L.N. Pfeiffer and K.W. West, Phys. Rev. Lett. 67 (1991) 3298; A. Schmeller, A.R. Goñi, A. Pinczuk, J.S. Reiner, J.M. Calleja, B.S. Dennis, L.N. Pfeiffer and K.W. West, Phys. Rev. B 49 (1994) 14778.

[2] W. Hansen, M. Horst, J.P. Kotthaus, U. Merkt. Ch. Sikorkski and K. Ploog, Phys. Rev. Lett. 58 (1987) 2586; T. Demel, D. Heitmann, P. Grambow and K. Ploog, Phys. Rev. B 38 (1988) 12732.

[3] B.Y.-K. Hu and S. Das Sarma, Phys. Rev. Lett. 68 (1992) 1750; Phys. Rev. B 48 (1993) 5469. See also S. Das Sarma and E.H. Hwang, preprint.

[4] J. Sólyom, Adv. Phys. 28 (1979) 201. For a fairly recent review, see e.g. in: The Many-Body Problem, ed. D.C. Mattis (World Scientific, Singapore, 1993).

[5] See the calculations by Das Sarma and co-workers [Ref. 3] for a detailed account of Fermi-liquid and Luttinger liquid comparison.

[6] K.S. Singwi, M.P. Tosi, R.H. Land and A. Sjölander, Phys. Rev. 179 (1968) 589.

[7] R.K. Moudgil, P.K. Ahluwalia and K.N. Pathak, Phys. Rev. B 51 (1995) 1575.
[8] S. Yarlagadda and G.F. Giuliani, Phys. Rev. B 40 (1989) 5432.

[9] A.K. Rajagopal and J.C. Kimball, Phys. Rev. B 15 (1977) 2819.

[10] W.I. Friesen and B. Bergersen, J. Phys. C 13 (1980) 6627; V.B. Campos, M.H. Degani and O. Hipōlito, Superlatt. Microstruct. 17 (1995) 85 .

[11] L. Calmels and A. Gold. Phys. Rev. B 51 (1995) 8426.

[12] P.F. Williams and A.N. Bloch, Phys. Rev. B 10(1974) 1097.

[13] G.Y. Hu and R.F. O'Connell, Phys. Rev. B 42 (1990) 1290

[14] Q.P. Li and S. Das Sarma, Phys. Rev. B 43 (1991) 11768

[15] R. Lobo, K.S. Singwi and M.P. Tosi, Phys. Rev. 186 (1969) 470.

[16] H. Yasuhara, Solid State Commun. 11 (1972) 1481; K Awa, H. Yasuhara and T. Asaki, Phys. Rev. B 25 (1982) 3670 .

[17] S. Nagano, K.S. Singwi and S. Ohnishi, Phys. Rev. B 29 (1984) 1209.

[18] A. Gold and A. Ghazali, Phys. Rev. B 41 (1990) 7626.

[19] S. Qin, S. Liang. Z. Su and Y. L.u. Phys. Rev. B 52 (1995) 5475 .

[20] F. Green, D. Neilson, L. Świerkowski. J. Szymański and D.J.W. Geldart, Phys. Rev. B 47 (1993) 4187.

[21] S. Tanaka and S. Ichimaru, Phys. Rev. B 39 (1989) 1036.

[22] R. Fantoni and M.P. Tosi, Physica B 217 (1996) 35. 